1 Early-stage aeolian protodunes: bedform development and sand transport dynamics

3

4 Matthew C. Baddock ${ }^{1}$, Joanna M. Nield ${ }^{2}$ and Giles F.S. Wiggs ${ }^{3}$

5

$6{ }^{1}$ Department of Geography, Loughborough University, Loughborough, LE11 3TU, 7 UK

$8{ }^{2}$ Geography and Environment, University of Southampton, Southampton, SO17 1BJ, 9 UK

${ }^{3}$ School of Geography and Environment, University of Oxford, Oxford, OX1 3QY, UK

*Corresponding author

Tel: $+44(0) 1509222798$

14 Fax: +44 (0)1509223930

15 Email: m.c.baddock@lboro.ac.uk

17 Abstract

18 Early-stage aeolian bedforms, or protodunes, are elemental in the continuum of dune 19 development and act as essential precursors to mature dunes. Despite this, we know 20 very little about the processes and feedback mechanisms that shape these nascent bedforms. Whilst theory and conceptual models have offered some explanation for

22 protodune existence and development, until now, we have lacked the technical 23 capability to measure such small bedforms in aeolian settings. Here, we employ 24 terrestrial laser scanning to measure morphological change at the high frequency 25 and spatial resolution required to gain new insights into protodune behaviour. On a $260.06 \mathrm{~m}$ high protodune, we observe vertical growth of the crest by $0.005 \mathrm{~m}$ in two 27 hours. Our direct measurements of sand transport on the protodune account for such 
growth, with a reduction in time-averaged sediment flux of $18 \%$ observed over the crestal region. Detailed measurements of form also establish key points of morphological change on the protodune. The position on the stoss slope where erosion switches to deposition is found at a point $0.07 \mathrm{~m}$ upwind of the crest. This finding supports recent models that explain vertical dune growth through an upwind shift of this switching point. Observations also show characteristic changes in the asymmetric cross section of the protodune. Flow-form feedbacks result in a steepening of the lee slope and a decline in lower stoss slope steepness (by $3^{\circ}$ ), constituting a reshaping of protodune form towards more mature dune morphology. The approaches and findings applied here, a) demonstrate an ability to quantify processes at requisite spatial and temporal scales for monitoring early-stage dune evolution, b) highlight the crucial role of form-flow feedbacks in enabling early-stage bedform growth, alluding to a fluctuation in feedbacks that require better representation in dune models, and c) provide a new stimulus for advancing understanding of aeolian bedforms.

1. Introduction

The initiation and early stages of aeolian dune development remain underresearched and poorly understood (Lancaster, 1996; Kocurek et al., 2010). From studies undertaken on mature bedforms, it is recognised that interactions and feedbacks between topography, boundary layer airflow and sediment transport are crucial in the growth and dynamic equilibrium of bedforms (Lancaster et al., 1996; Wiggs et al., 1996; Walker and Nickling, 2002). One of the key hindrances to understanding the behaviour of early-stage bedforms is the limited influence these small and growing features have on airflow, so that the depth of the layer where flow is altered by the bedform is very thin. This makes it extremely challenging to measure relevant flow properties (e.g. McKenna Neuman et al., 1997; Claudin et al., 2013). The near-surface internal boundary layer is a crucial region, however, as it is within this layer that shear stress variation caused by the intrusion of the bedform into the flow is significant, so that shear measured here is most relevant to sand transport at the surface (Mulligan, 1988; Frank and Kocurek, 1996; Lancaster et al., 1996; Wiggs et al., 1996; Wiggs, 2001; Walker and Nickling, 2002; Weaver and Wiggs, 2011). 
60

Some of the fundamental concepts of early-stage dune development were first investigated in detail by the landmark study of Kocurek et al. (1992). Their work examined the complete development of aeolian bedforms and identified a series of morphological stages ranging from irregular sand patches (Stage 1) through to fully developed dune forms (Stages 4,5 ) based on observations made at Padre Island, Texas, USA (Figure 1). Bedforms at Stage 1 are essentially disorganised patches of sand (a few centimetres in height) that have started to migrate away from a sand nucleation site, but do not display any characteristic elements of dune form. Bedforms that have developed to Stage 2, those termed "wind-ripple protodunes" by Kocurek et al. (1992), are small features that are not mature enough to demonstrate grainfall or grainflow behaviour in their lee, but do possess an identifiable crest. In planform, Stage 2 features have also begun to develop towards recognisable barchanoid shapes but their cross sections may show reversed asymmetry with respect to mature dune forms i.e., a steeper stoss slope than lee (Cooper, 1958; Hesp and Arens, 1997; Nield et al., 2011). Kocurek et al. (1992) also established that Stage 2 bedforms typically produce a slight modification of the near-surface wind speed (e.g. Claudin et al., 2013). While Stage 1 and 2 forms are an essential part of the aeolian bedform continuum it is particularly difficult to understand the morphodynamic processes that drive their development because these forms are small ( $<0.35 \mathrm{~m}$ in height), migrate rapidly, and can be highly ephemeral in nature. Without appropriate wind and sand supply conditions, such bedforms are likely to degrade over timescales of hours to days (Lancaster, 1996; Nield, 2011).

Despite the challenges inherent in measuring the flow-form dynamics of Stage 2 bedforms, these features demand research attention because of their widespread appearance in both dryland and coastal aeolian environments and their importance as precursors of fully-developed dunes as well as, ultimately, in the development of dune patterns (Kocurek et al., 2010). Given the difficulties of undertaking flow measurements, a range of theoretical, numerical and physically-based modelling studies have been used in the study of bedform development (e.g. Sauermann et al., 2001; Andreotti et al., 2002; Groh et al., 2009; Durán et al., 2010). The majority of these studies have considered the evolution of a pre-existing pile of sand, and specifically the role of saltation saturation length in understanding minimal dune size 
92 and the prediction of whether a dune grows or shrinks (Hersen et al., 2002; Parteli et al., 2007).

94 The relative absence of process studies focusing on early-stage protodunes since 95 the work of Kocurek et al. (1992) is testament to the difficulties of observation. While some morphological assessment of early bedforms (Stage 3 ) has been undertaken (e.g. Elbelrhiti, 2012), difficulties in measuring flow, sediment transport and surface change at high temporal and spatial resolutions (Claudin et al., 2013) mean that linkages between bedform dynamics and sand transport patterns over early-stage dunes have not yet been fully established.

101 The advent of new field technologies has provided a significant opportunity to overcome the difficulties associated with high-resolution measurement of aeolian processes, providing a stimulus for the study of protodune dynamics and development. In particular, terrestrial laser scanning (TLS) offers a valuable capability for the quantification of surface change at high temporal and spatial resolution while being non-invasive to the airflow (Nield et al., 2011; 2017). The aim of the work described here was to use repeat TLS and sand flux measurements to provide the first high-resolution coupled observations of sand transport on, and morphological development of, aeolian protodunes. The novel approach used here provides fresh insights into the morphodynamics of what have remained poorly understood early-stage aeolian bedforms.

\section{2. Methods}

113 Brancaster beach on the North Norfolk (UK) coastline has a wide sandy beach

114 orientated east-west and is backed by low dunes and salt marsh (May and Hansom, 115 2003). During low tide and with drying, alongshore winds, the fetch and sand 116 availability in these conditions of reduced surface moisture afford conditions 117 conducive for sand strips to form, features that are classified as Stage 2 protodunes 118 by Kocurek et al. (1992). On the 12th August 2016 a migrating protodune on this 119 beach was monitored for around 2.5 hours from 16:30 BST. High tide was at 120 approximately $13: 45$, and average temperature and relative humidity during the 121 experiment was $24.4^{\circ} \mathrm{C}$ and $52.8 \%$ respectively. The study protodune was 122 approximately $0.06 \mathrm{~m}$ high (Figure 2), $3 \mathrm{~m}$ long, $2.6 \mathrm{~m}$ wide, displaying a steeper 123 stoss than lee slope, and was positioned within a field of other protodunes 
124 developing on the beach. Mean grain diameter of sand on the protodune was 237

$125 \mu \mathrm{m}$.

126 Near-surface wind speed was recorded at a frequency of $1 \mathrm{~Hz}$ at $0.62 \mathrm{~m}$ height using

127 a Gill 2D Windsonic anemometer positioned $2.8 \mathrm{~m}$ upwind and $0.3 \mathrm{~m}$ offset from the

128 protodune. Wenglor optical gate sensors have emerged as useful devices for

129 monitoring high-frequency saltation (e.g. Hugenholtz and Barchyn, 2011; Davidson-

130 Arnott et al. 2012). Four Wenglor sensors provided saltation measurements at points

131 upwind, on top of, and downwind of the protodune (Figure 2), sampling at $1 \mathrm{~Hz}$ and

132 positioned $0.02 \mathrm{~m}$ above the surface. The height of the Wenglors was adjusted as

133 the protodune migrated (+/- $0.01 \mathrm{~m})$. Wenglor counts were converted to a mean flux

134 using the methods of Barchyn et al. (2014), for each scan interval of approximately 4

135 minutes.

136 Surface topography was measured using a Leica P20 Scanstation TLS with

137 instrument resolution set to a horizontal point spacing of $1.6 \mathrm{~mm}$ at a distance of 10

$138 \mathrm{~m}$. The TLS was approximately 8-10 $\mathrm{m}$ from the bedform and remained in position

139 for the duration of the experiment. The protodune was rescanned approximately

140 every 4 minutes and produced mean point densities of 84,200 points $/ \mathrm{m}^{2}$.

141 In addition to determining surface topography, the TLS was also used to give an

142 indication of surface moisture and an independent measure of saltation activity. The

143 raw point cloud was filtered to separate surface and saltation partial returns using the

144 methods of Nield and Wiggs (2011). Surface point return intensities within $0.01 \mathrm{~m}$

145 grid squares were converted to surface moisture using the approach of Nield et al.

146 (2011) using $W=5.22 \times 10^{-6} S^{-6.43}$ where $W$ is the gravimetric moisture content (as a

147 percentage) and $S$ is the TLS return signal intensity derived from the P20 TLS. The

148 percentage of the study area where saltation was detected above the surface (with

$1490.01 \mathrm{~m}$ grid resolution) signifies a proxy for system-wide transport (Figure 3b).

150 The topography data for the protodune were de-trended for the large scale beach

151 slope by fitting a surface through all points representing the interstrip area,

152 determined as those that did not change throughout the experiment. The migration

153 rate of the protodune was calculated by cross-correlation of the cross section form at

154 each time interval (Figure 3a). These migration rates were used to horizontally back-

155 shift cross sections at different times, thereby allowing any change in the topographic 
156 shape of the protodune to be highlighted independently of migration rate. Surface

157 change between time intervals along each cross section was calculated by first

158 smoothing the surface using a $0.31 \mathrm{~m}$ moving window filter to reduce the influence of

159 surface ripples (wavelengths 0.06-0.2 $\mathrm{m}$ ) on the calculations. The overall mean

160 difference in height between smoothed and unsmoothed data was $-5.8 \times 10^{-6} \mathrm{~m}$.

161 To assess the combined errors of the instrument and post-processing methods, we 162 analysed a $1.5 \times 0.3 \mathrm{~m}$ section of the beach upwind of the protodune at a horizontal 163 resolution of $0.01 \times 0.01 \mathrm{~m}$. This grid size implicitly defines the horizontal error as $1640.01 \mathrm{~m}$. The mean absolute vertical change with respect to the stable beach surface 165 was $5.5 \times 10^{-4} \mathrm{~m}$ with a standard deviation of $6.8 \times 10^{-4} \mathrm{~m}$. For change detection 166 measured over a 4 minute period this corresponds to a surface change rate mean 167 error of $2.29 \times 10^{-6} \mathrm{~m} / \mathrm{s}$.

168 3. Results

169 For the 2.5 hours of the experiment, wind speed was relatively consistent, varying 170 between 5.5 and $6.5 \mathrm{~m} / \mathrm{s}$ (Figure 3a) and exceeding the critical threshold for 171 transport on the protodune as evidenced by the Wenglor data (Figure 3b). The 172 saltation activity, as measured independently by the Wenglors and by detection of 173 the spatial extent of saltation from the TLS, showed a positive relationship with wind 174 speed over the study period ( $R^{2}$ of 0.84 and 0.61 respectively; Figure $3 b$ ).

175 Throughout the experiment, the mean surface moisture content was $1.5 \%$ and the 176 extent of the surface area which demonstrated a change in moisture was $<1.4 \%$, 177 indicating that moisture did not fluctuate and was not a significant control on sand 178 flux or protodune dynamics. The wind direction was consistent throughout the 179 experiment (standard deviation $3.7^{\circ}$ ) and ranged from $242^{\circ}$ to $254^{\circ}$ with a mean 180 value of $249^{\circ}$. In the subsequent analyses, the protodune surface was orientated 181 parallel to the mean wind direction (and bedform migration direction). This facilitated 182 the quantification of cross section change parallel to the wind direction.

183 The general pattern of sand transport over the protodune, as expressed by Wenglor 184 flux at different positions on the bedform (W1, W2) over each 4 minute period and 185 normalised by the upwind flux (W0), is shown in Figure 4a. In this plot the locations 186 of normalised sand flux measurement are presented as distance from the upwind 187 protodune toe $(x=0)$ which, because the protodune was migrating, means that the 
horizontal positions of the Wenglor data show an upwind shift over time. The mean location of the protodune crest over the entire experiment was $0.99 \mathrm{~m}$ downwind from the toe $(x=0.99 \mathrm{~m})$ (Figure 4a). Given that the position of W1 shifted from $x=$

1910.82 to $0.26 \mathrm{~m}$, measurements at $\mathrm{W} 1$ represent transport in the zone upwind of the

192 crest for the whole experiment. Flux in this region upwind of the crest shows a

193 consistent increase with distance from the toe. The maximum observed flux (at $x=$

$1940.43 \mathrm{~m}$ ) was just over twice that measured upwind of the protodune (W0). Point W2

195 remained downwind of the crest throughout the period of monitoring, shifting from $\mathrm{x}=$

1962.47 to $1.81 \mathrm{~m}$, and the relative flux here was seen to decrease with increasing

197 distance from the toe (Figure 4a). This decrease followed the down-sloping

198 topography on the leeside.

199 For the duration of the experimental period, the mean rate of surface change, as 200 determined from bi-sequential TLS scans (i.e. the difference over approximately 8 201 minutes), shows a dominance of negative values (erosion) in the toe region, with a 202 maximum in erosion occurring at $x=0.21 \mathrm{~m}$ (Figure $4 b$ ). Downwind from here, the 203 rate of surface change became closer to 0 until it switched from net negative 204 (erosion) to net positive (deposition) at $\mathrm{x}=0.92 \mathrm{~m}$, a point just upwind of the dune 205 crest, $x=0.99 \mathrm{~m}$. Downwind from the crest, the rate of positive surface change reached a maximum at $x=2.0 \mathrm{~m}$ (1.01 $\mathrm{m}$ downwind from the crest), after which it declined towards negligible values near the downwind edge of the protodune.

While Figure 4 generalises about the patterns of transport and mean surface change, our data also enable us to examine how the protodune morphology changed over time as it migrated. Cross sections of the protodune at three different periods of the experiment are shown in Figure $5 \mathrm{a}$ (with cumulative migration rate in Figure $3 \mathrm{a}$ ). By transposing the profiles for these separate time periods, the changing shape of the protodune can be highlighted (Figure 5b). Here, it is shown that during the first hour

214 of the experiment the protodune increased in height by as much as $0.004 \mathrm{~m}$ in the 215 vicinity of the crest. In the second hour, however, the protodune largely retained its overall shape and height increased only by $0.001 \mathrm{~m}$, with the majority of change occurring on the lee slope, as represented by both deposition on the upper lee slope and erosion on the lower lee slope. Figure $5 b$ also reveals that through the course of the experiment, the length of the lee slope reduced by a distance of $0.5 \mathrm{~m}$ from its initial position at $x=3.4$ to $2.9 \mathrm{~m}$ after the second hour. These morphological 
221 changes are reflected in the distribution of net surface change observed in the

222 different time periods (Figure 5c). In the first hour of measurement there was a

223 strong dominance of positive vertical change around the crest area (between $\mathrm{x}=$

2240.34 and $1.75 \mathrm{~m}$ ) with a maximum rate of $0.051 \mathrm{~mm} / \mathrm{s}$. Positive surface change

225 during the second hour had a reduced maximum magnitude $(0.023 \mathrm{~mm} / \mathrm{s})$ and did

226 not extend as far downwind of the crest (occurring between $x=0$ and $1.34 \mathrm{~m}$ ).

227 Throughout both hours of measurement, reworking of the stoss and lee slopes is

228 evidenced by alternating negative and positive change between time periods,

229 emphasised in Figure 5c. The maximum slope angles throughout the duration of the

230 experiment vary between 13 and $21^{\circ}$ on the stoss, and $2.8-3.4^{\circ}$ on the leeside.

231 To investigate the sand transport patterns associated with the change in protodune

232 morphology, Figure 5d provides comparisons of the mean flux measured at W1 and

233 W2 (i.e. across the crest region), averaged during the first ( $\left.t_{1}\right)$ and second ( $\left.t_{2}\right)$ hours,

234 and overall for the whole experiment (Figure $5 \mathrm{~d}$ ). In the first hour, there was a

235 decrease in the mean normalised flux from 1.68 at $\mathrm{W} 1_{\mathrm{t} 1}$ to 0.81 at $\mathrm{W} 2_{\mathrm{t} 1}$ (Figure $5 \mathrm{~d}$ ),

236 corresponding to accretion across the crestal region (between $\mathrm{x}=0.34$ and $1.75 \mathrm{~m}$ )

237 (Figure $5 \mathrm{c}$ ). In the second hour, flux was $50 \%$ greater at $\mathrm{W} 2 \mathrm{t} 2$ than $\mathrm{W}_{1 \mathrm{t} 2}$, indicating

238 an erosive potential between these points. While some accretion is evident in the

239 second hour from the toe $(x=0 \mathrm{~m})$ to $x=1.35 \mathrm{~m}$, the erosive potential of the

240 increasing flux is reflected in negative surface change measured between $x=1.35$

241 and $1.74 \mathrm{~m}$ (Figure 5c). The overall mean flux over the duration of the experiment

242 indicates a reduction in transport (18\%) between W1 and W2 (Figure 5d). This is

243 reflected by the overall net accretion of sand in the protodune which is focused in the

244 crest region (Figure 5b,c).

245 4. Discussion

246 Given the sparse amount of both process and morphological observations on early-

247 stage bedforms, our findings provide the first fully quantitative data on sand transport

248 patterns over a Stage 2 protodune. Kocurek et al (1992) reported a slight increase in

249 wind velocity on the stoss slope of these features, and specified that on the leeside

250 wind speeds were reduced to $15-80 \%$ of the velocity at the crest. Although our study

251 did not seek to quantify flow over the bedform directly, the broad airflow patterns are

252 reflected in our transport measurements which show an increase in flux up the stoss 
253 slope, offering evidence for the micro-topographically driven acceleration of flow

254 (Kocurek et al., 1992). On the leeside, the observed trend of decreasing transport

255 (Figure 4a), with a reduction in time-averaged flux between the stoss and lee slope

256 (Figure 5d), is indicative of flow deceleration downwind of the crest. The angles of

257 the stoss and lee slopes on our protodune are also in line with measurements made

258 on similar bedforms, including stoss slopes steeper than lee slopes (Hesp and Arens,

259 1997) and overall leeside angles comparable to Stage 2 features on Padre Island

260 which were typically $<10^{\circ}$ (Kocurek et al., 1992).

261 The geomorphic interest concerning Stage 2 protodunes is that they are the pre-

262 cursor bedforms for mature dunes. For protodunes to develop into barchan or

263 transverse bedforms there is a requirement that they grow in height and also

264 undergo reshaping to a form more characteristic of fully formed dunes (e.g.

265 Lancaster, 1985; Tsoar, 1985; Elbelrhiti, 2012). For such crestal growth to occur, the

266 transition from a net eroding surface to a net depositional surface (the point where

267 sand flux is at a maximum) must occur upwind of the crest (Andreotti et al., 2002a,b;

268 Claudin et al. 2013). A crucial result for the explanation of the vertical development

269 of bedforms is the evidence we provide here of the location where surface change

270 (or erosion rate) switches from erosion to deposition in relation to the crest. In terms

271 of our sand flux measurements, while we see a time-averaged overall reduction of

272 transport by $18 \%$ between locations upwind and downwind of the crest (Figure $5 \mathrm{~d}$ ),

273 the transition from a net eroding to a net depositional surface is impossible to locate

274 precisely from the flux measurements because of the limited spatial resolution of the

275 Wenglor instruments. However, from the TLS topographic data, and analysing for the

276 mean surface topography over the entire measurement period (at a horizontal grid

277 resolution of $0.01 \mathrm{~m}$ ), it is clear that the location of this switch from erosion to

278 deposition occurs at a distance which is $0.07 \mathrm{~m}$ upwind of the crest. The flat nature

279 of the topography in the crestal region of the protodune makes it difficult to identify

280 the exact position of the crest, even with the very high resolution of the TLS

281 instrument. However, given the known vertical error for the TLS measurements of

$2825.5 \times 10^{-4} \mathrm{~m}$, analysis of all scans revealed a mean error in the horizontal positioning

283 of the crest of only $0.02 \mathrm{~m}$, and skewed further downwind from the crest identified

284 from the TLS data. This indicates that the $0.07 \mathrm{~m}$ upwind shift (relative to the crest)

285 that we have identified for the location of the switch from erosion to deposition 
represents a conservative value, and it is in agreement with the data of Claudin et al.

287 (2013) investigating larger dune bodies. Our surface change measurements over the duration of the experiment also establish that the upwind position of this switch does indeed result in vertical growth in the crest region (Figure 5b, c).

290 For an emerging protodune to develop beyond a Stage 2 form, it must not only grow 291 vertically but also change in cross-sectional shape (Lancaster, 1985; Kocurek et al., 292 1992). Until now the characteristics of such morphological change have not been possible to measure in sufficient temporal and spatial detail on a bedform of this small size. However, by utilising high frequency TLS measurements of surface change, and combining these with the rapid migration rate of protodunes, we are now in a position to examine this dynamic change in morphology. Despite the relatively short period of observation, we identify two characteristic elements of form change occurring on the protodune. Firstly, we see evidence of the lee slope steepening as a result of the $0.5 \mathrm{~m}$ reduction in its length over the course of the monitoring period (Figure 5b). Such an observation is significant because this steepening of the lee must occur for evolution to the next stage of bedform, the grainfall protodune, and subsequently, for airflow to become separated from the surface (Kocurek et al., 1992) (Figure 1). Secondly, the irregular form observed at the start of the experiment on the lower stoss slope also undergoes change (Figure $5 a$ ). During the first hour, erosion was observed in the region $x=0$ to $0.34 \mathrm{~m}$ (Figure 5c), which resulted in a smoother stoss slope. The change in the lower stoss slope is demonstrated by a $3^{\circ}$ reduction in the mean slope angle between $x=0.10$ and 0.25 $\mathrm{m}$, averaged over the course of the experiment. Confidence in determining stoss angle change is highest in the region $x=0.10 \mathrm{~m}$ to $0.25 \mathrm{~m}$ because ripples, whose presence can confound small scale angle quantification, were absent here. This observed change in angle on both the lee and lower stoss slopes provides evidence for a reduction in the reverse asymmetry (positive skew) of the protodune form, and shows a development of these slopes towards angles more typical of mature dune forms (Tsoar, 1985; Kocurek et al., 1992).

In the crest region, the general pattern of accretion conforms to models explaining dune growth (Andreotti et al. 2002; Claudin et al., 2013) but the pattern of crestal

317 change is not constant, and its rate varies over the course of the experiment (Figure $3185 b, c$ ). Over the highest third of the bedform (between $x=0.34$ and $1.75 \mathrm{~m}$ ) vertical 
319 accretion is most apparent during the first hour. Through the second hour there is

320 little change in form of the crestal area such that airflow and sand transport must

321 maintain form in this region. This observed variability in crest growth and form

322 change highlights sensitivity in protodune development, likely due to variable flow-

323 form feedbacks or boundary conditions (e.g. sand supply) whose influence may not

324 be accounted for in generalised models.

325 On mature dunes, reworking of the lee slope is dominated by grainfall and grainflow 326 processes (Nickling et al., 2002; Nield et al., 2017), neither of which occur on under327 developed Stage 2 bedforms. Instead, Kocurek et al. (1992) hypothesize that leeside 328 morphological change on protodunes is driven by differences in rates of sand 329 transport caused by flow expansion and deceleration, which we observe through 330 decreased flux rates on the leeside (Figure 4a). However, over bedforms with limited 331 topographic signature, variations in transport may only be very subtle. Where aeolian 332 studies of flow over Stage 2 bedforms are lacking, useful interpretations can be 333 obtained from fluvial studies of low-angle bedforms. In investigating a subaqueous 334 form with a maximum lee slope of $14^{\circ}$, Best and Kostaschuk (2002) identified that 335 flow in the leeside, which expanded but remained attached to the surface, could generate highly turbulent horizontal and vertical velocities. For aeolian protodunes, such variability in flow may help drive the highly inconsistent magnitudes of accretion and deflation we observed downwind of $x=1.75 \mathrm{~m}$, representing the lee area and further downwind (Figure 5c).

\section{Conclusion}

341 Studies of early-stage bedforms are rare in aeolian geomorphology. Part of the 342 difficulty concerning their investigation relates to the fact their small size makes them 343 highly morphologically dynamic bedforms that exhibit limited intrusion into the boundary layer. Novelty is required therefore in the experimental monitoring and data analysis approaches that can bring about improved understanding of these features. The dataset presented here demonstrates an ability to represent linked aspects of

347 both process and bedform change, in space and time, at scales commensurate with 348 protodune development.

349 In this study, we used terrestrial laser scanning to achieve a highly detailed 350 quantification of protodune form change, and link this to the first measurements of 
351 sand transport over this type of bedform. We provided evidence of both vertical 352 growth of the protodune and the reworking of its shape, two ingredients necessary 353 for the development of mature dunes from early-stage bedforms. We explain the 354 observed changes in form through a series of key process findings. In particular, we identify that a switch from erosion to deposition occurs at a point $(0.07 \mathrm{~m})$ upwind of the protodune crest. The role played by the positioning of this switch has emerged as a critical argument for explaining aeolian bedform growth. Here, we provide evidence of the existence of the upwind shift of erosion to deposition on one of the smallest aeolian bedforms, and we can relate this point to observations that the protodune was undergoing vertical crestal growth. The pattern of sand transport and the $18 \%$ reduction in time-averaged sediment flux between upwind and downwind of the crest also reveal the deposition potential at the crest region over the duration of the experiment.

364 Rapid topographic monitoring has identified the focal points where morphological 365 change occurred as the protodune developed. Over the irregular and asymmetric form of the protodune, characterised by a stoss slope steeper than the lee, the shortening of the leeside length indicates a steepening of that slope over the experimental period, while smoothing and a reduction in slope by $3^{\circ}$ occurred on the lower stoss slope. These changes in the lee and stoss slopes toward those more characteristic of a mature dune reveal a reduction in overall protodune asymmetry.

371 On the lee slope, as the protodune is at a stage of development where grainfall 372 processes do not yet operate, high variability in rates of net surface change may be

373 demonstrative of highly changeable sand transport driven by turbulent velocity 374 components, as found on fluvial low-angle dune proxies.

375 Aside from the novel quantifications of aeolian process provided by this study, the 376 wider potential of the techniques and approaches used here for the testing of 377 theoretical explanations of development of early-stage bedforms is also illustrated. In 378 offering the means to carry out measurements of process form change at the 379 requisite scales, methods are established that will assist in tackling some of the most 380 persistent uncertainties in dune morphodynamics. 
Data processing used IRIDIS Southampton Computing Facility. We thank E. Callicott for field assistance and D. Beben, T. Bishop, L. Riddy and W. Nickling for instrument construction and calibration. Detailed comments from S. Lane, the Associate Editor, and reviewers $G$. Kocurek and A. Baas are much appreciated for helping to improve the manuscript.

References

391

Andreotti B, Claudin P, Douady S. 2002a. Selection of dune shapes and velocities: part 1: dynamics of sand, wind and barchans. European Physical Journal B 28: 321393339.

394 Andreotti B, Claudin P, Douady S. 2002b. Selection of dune shapes and velocities: 395 Part 2: A two-dimensional modelling. European Physical Journal B 28: 341-352. Barchyn TE, Hugenholtz CH, Li B, McKenna Neuman C, Sanderson RS. 2014. From particle counts to flux: Wind tunnel testing and calibration of the 'Wenglor' aeolian 398 sediment transport sensor. Aeolian Research 15: 311-318, 399 DOI:10.1016/j.aeolia.2014.06.009.

400 Best J, Kostaschuk R. 2002. An experimental study of turbulent flow over a low401 angle dune. Journal of Geophysical Research-Oceans 107: 3135, 402 DOI:10.1029/2000JC000294.

403 Claudin P, Wiggs GFS, Andreotti B. 2013. Field evidence for the upwind velocity shift 404 at the crest of low dunes. Boundary-Layer Meteorology DOI:10.1007/s10546-013405 9804-3.

406 Cooper WS. 1958. Coastal sand dunes of Oregon and Washington. Geological 407 Society of America Memoir, 72, $167 \mathrm{pp}$.

408 Davidson-Arnott RGD, Bauer BO, Walker IJ, Hesp PA, Ollerhead J, Chapman C. 409 2012. High-frequency sediment transport responses on a vegetated foredune. Earth 410 Surface Processes and Landforms 37: 1227-1241, DOI:10.1002/esp.3275.

411 Duran O, Parteli EJR, Herrmann HJ. 2010. A continuous model for sand dunes: 412 Review, new developments and application to barchan dunes and barchan dune 413 fields. Earth Surface Processes and Landforms 35: 1591-1600. 
414 Elbelrhiti H. 2012. Initiation and early development of barchan dunes: a case study of 415 the Moroccan Atlantic Sahara desert. Geomorphology 138: 181-188,

416 DOI10.1016/j.geomorph.2011.08.033.

417 Frank A, Kocurek G. 1996. Airflow up the stoss slope of sand dunes: limitations of 418 current understanding. Geomorphology 17: 47-54.

419 Groh C, Rehberg I, Kruelle CA. 2009. How attractive is a barchan dune? New 420 Journal of Physics, 11: 023014.

421 Hersen P, Douady S, Andreotti B. 2002. Relevant length scale of barchan dunes. 422 Physical Review Letters 89(26): 264301.

423 Hesp PA, Arens SM. 1997. Crescentic dunes at Schiermonnikoog, The Netherlands. 424 Earth Surface Processes and Landforms 22: 785-788.

425 Hugenholtz $\mathrm{CH}$, Barchyn TE. 2011. Laboratory and field performance of a laser 426 particle counter for measuring aeolian sand transport. Journal of Geophysical 427 Research 116: F01010, DOI:10.1029/2010F001822.

428 Kocurek G, Townsley M, Yeh E, Havholm K, Sweet ML. 1992. Dune and dune-field 429 development on Padre Island, Texas, with implications for interdune deposition and 430 water-table-controlled accumulation. Journal of Sedimentary Petrology 62: 622-635.

431 Kocurek G, Ewing RC, Mohrig D. 2010. How do bedform patterns arise? New views 432 on the role of bedform interactions within a set of boundary conditions. Earth Surface 433 Processes and Landforms 35: 51-63, DOI: 10.1002/esp.1913.

434 Lancaster N. 1985. Variations in wind velocity and sand transport on the windward 435 flanks of desert sand dunes. Sedimentology 32: 581-593.

436 Lancaster N. 1996. Field studies of sand patch initiation processes on the northern 437 margin of the Namib Sand Sea. Earth Surface Processes and Landforms 21: 947438954.

439 Lancaster N, Nickling WG, McKenna Neuman CK, Wyatt VE. 1996. Sediment flux 440 and airflow on the stoss slope of a barchan dune. Geomorphology 17: 55-62.

441 May VJ, Hansom JD. 2003. Coastal Geomorphology of Great Britain. Joint Nature 442 Conservation Committee: Peterborough; 737 pp.

443 McKenna Neuman CK, Nickling WG, Lancaster N. 1997. Relations between dune 444 morphology, airflow, and sediment flux on reversing dunes, Silver Peak, Nevada. 445 Sedimentology 44: 1103-1113.

446 Mulligan K. 1988. Velocity profiles measured on the windward slope of a transverse 447 dune. Earth Surface Processes and Landforms 13: 573-582. 
448 Nickling WG, McKenna Neuman CM, Lancaster N. 2002. Grainfall processes in the 449 lee of transverse dune, Silver Peak, Nevada. Sedimentology 49: 191-209, 450 DOI:10.1046/j.1365 -3091.2002.00443.x.

451 Nield JM. 2011. Surface moisture-induced feedback in aeolian environments.

452 Geology 39: 915-918, DOI: 10.1130/G32151.1.

453 Nield JM, Wiggs GFS. 2011. The application of terrestrial laser scanning to aeolian 454 saltation cloud measurement and its response to changing surface moisture. Earth 455 Surface Processes and Landforms 36: 273-278, doi:10.1002/esp.2102.

456 Nield JM, Wiggs GFS, Squirrel RS. 2011. Aeolian sand strip mobility and protodune 457 development on a drying beach: examining surface moisture and surface roughness 458 patterns measured by terrestrial laser scanning. Earth Surface Processes and 459 Landforms 36: 513-522, DOI: 10.1002/esp.2071.

460 Nield JM, Wiggs GFS, Baddock MC and Hipondoka MRT. 2017. Coupling leeside 461 grainfall to avalanche characteristics in aeolian dune dynamics. Geology 45: 271-274, 462 DOI:10.1130/G38800.1.

463 Parteli EJR, Duran O, Herrmann HJ. 2007. Minimal size of a barchan dune. Physical 464 Review E 75:011301.

465 Sauermann G, Kroy K, Herrmann HJ. 2001. Continuum saltation model for sand 466 dunes. Physical Review E 64: 031305.

467 Tsoar H. 1985. Profile analysis of sand dunes and their steady state significance. 468 Geografiska Annaler A 67: 47-59.

469 Walker IJ, Nickling WG. 2002. Dynamics of secondary airflow and sediment 470 transport over and in the lee of transverse dunes. Progress in Physical Geography 471 26: 47-75, DOI: 10.1191/0309133302pp325ra.

472 Wiggs GFS. 2001. Desert dune processes and dynamics. Progress in Physical 473 Geography 25: 55-81.

474 Weaver CM, Wiggs GFS. 2011. Field measurements of mean and turbulent airflow 475 over a barchan sand dune. Geomorphology 128: 32-41, DOI:

476 10.1016/j.geomorph.2010.12.020.

477 Wiggs GFS, Livingstone I, Warren A. 1996. The role of streamline curvature in sand 478 dune dynamics: evidence from field and wind tunnel measurements. Geomorphology 479 17: 29-46. 


\section{Stage 1: Irregular sand patch or strip}

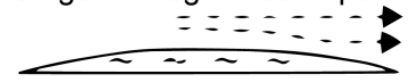

Stage 2: Protodune with wind-ripples and reverse asymmetry

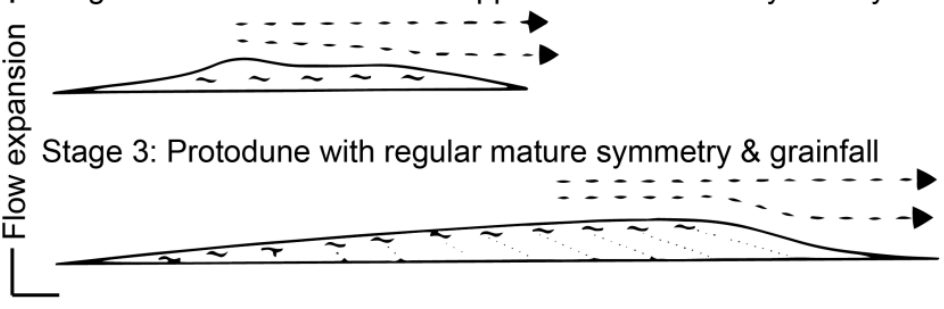

Stage 4: Slipface-less protodune with grainfall

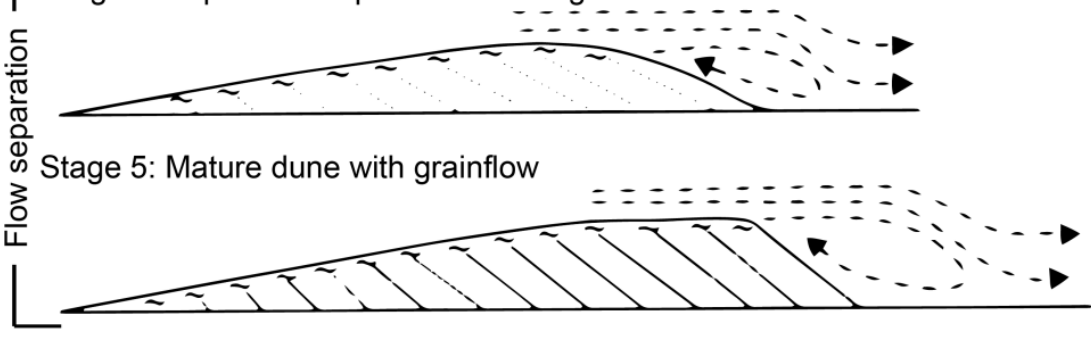

480

481 Figure 1: The five stages of dune development and their leeside airflow patterns as 482 presented by Kocurek et al. (1992). 

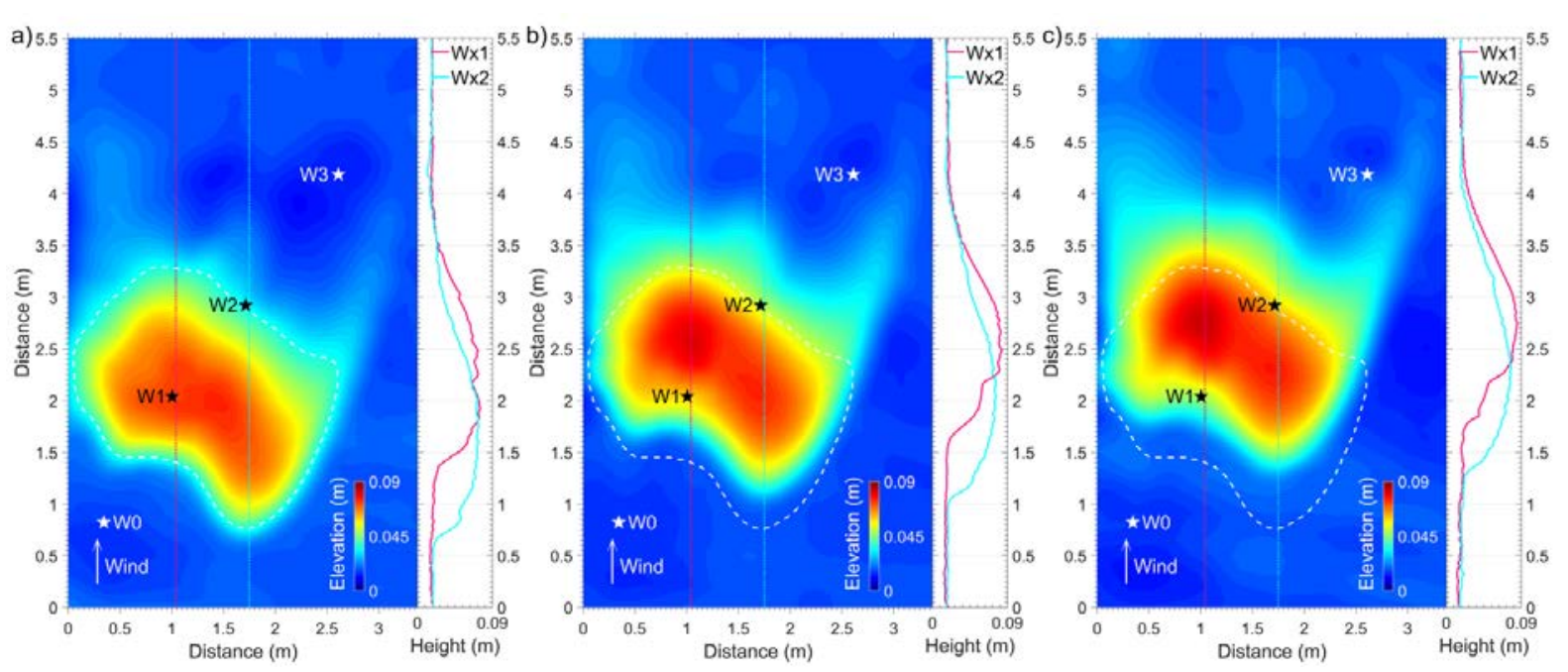

485 Figure 2: DEMs and cross sections of the protodune form at a) the start, b) after the 486 first hour and c) after the second hour. Wenglor (sand transport) sensor placements 487 and related cross section transects are also indicated. The protodune was migrating 488 in line with the wind direction. White dashed line in b) and c) is the base of the 489 protodune at the start to illustrate migration. Data have been smoothed using a 0.30 $490 \mathrm{~m}$ filter to remove the ripples and show changes in protodune form. 

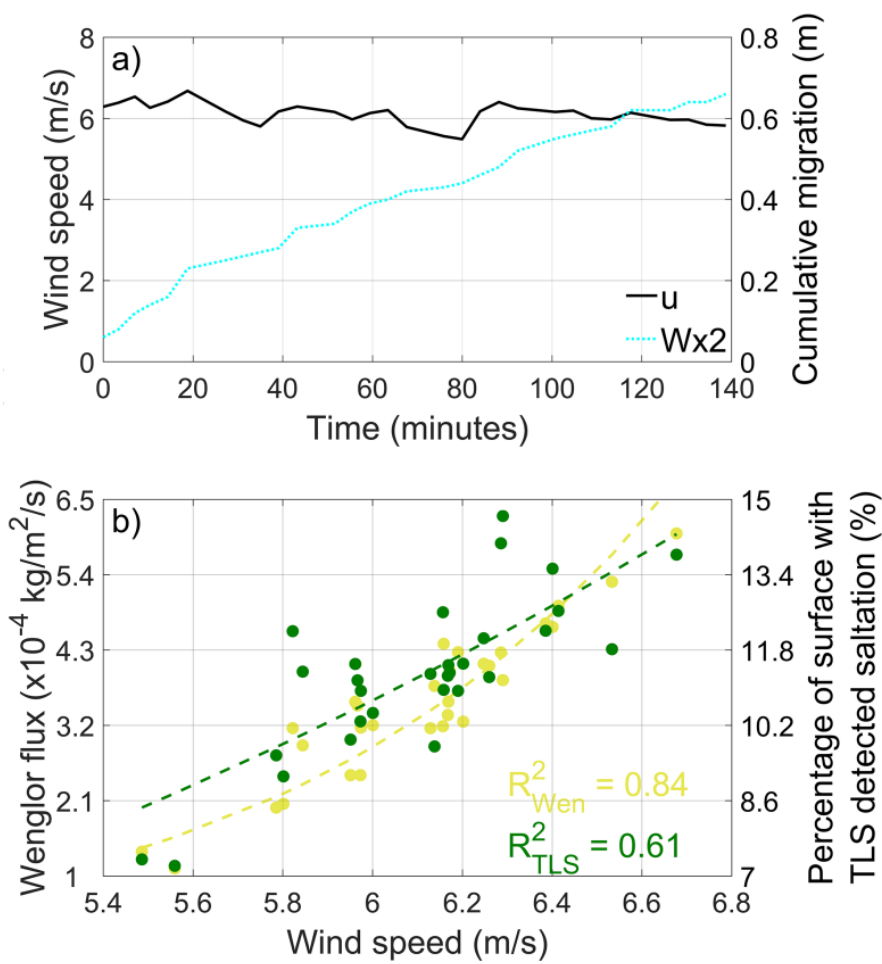

492

493 Figure 3: a) Upwind wind speed and cumulative migration distance of the Wx2 cross 494 section over the experiment duration, showing a consistent migration rate over time. 495 b) Mean saltation flux from all Wenglors (W0-W3) at $0.02 \mathrm{~m}$ height, and percentage 496 of the measurement area where saltation was detected by the TLS. Each of these 497 transport indicators shows a positive relationship between wind speed and flux. 

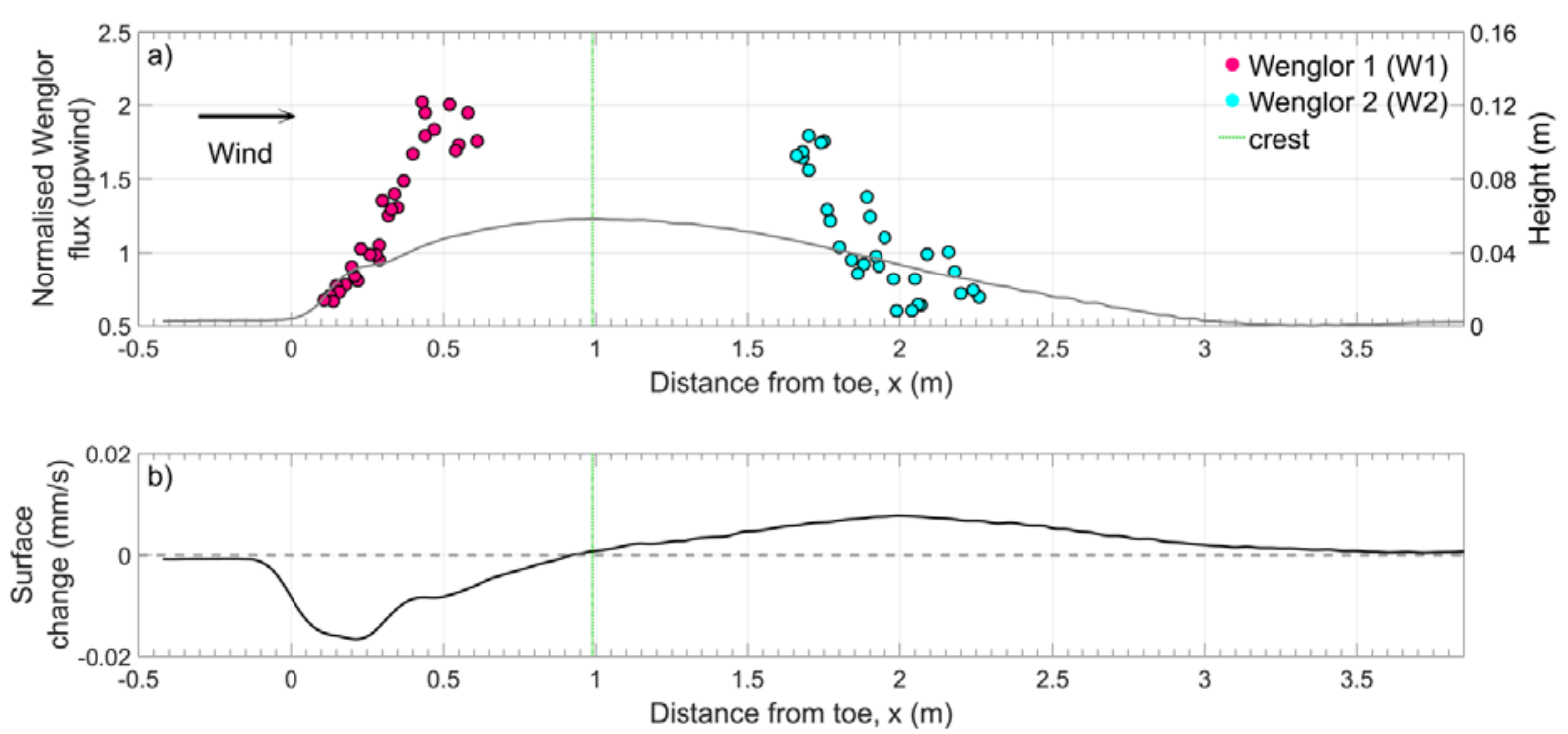

500 Figure 4: a) Transport pattern over the time-averaged protodune form. Wenglor

501 fluxes at W1 and W2 have been normalised to the upwind flux at W0 (-0.6 $\mathrm{m}$ from

502 the toe) to account for wind speed variation through time. Topography (grey) is the

503 time-averaged Wx2 cross section of Figure 2. b) Mean rate of absolute surface

504 change over the entire experiment, as derived from differences between bi-

505 sequential TLS scans. The point where the mean absolute surface change switches

506 from erosion to deposition is located approximately $0.07 \mathrm{~m}$ upwind of the mean crest 507 location. 

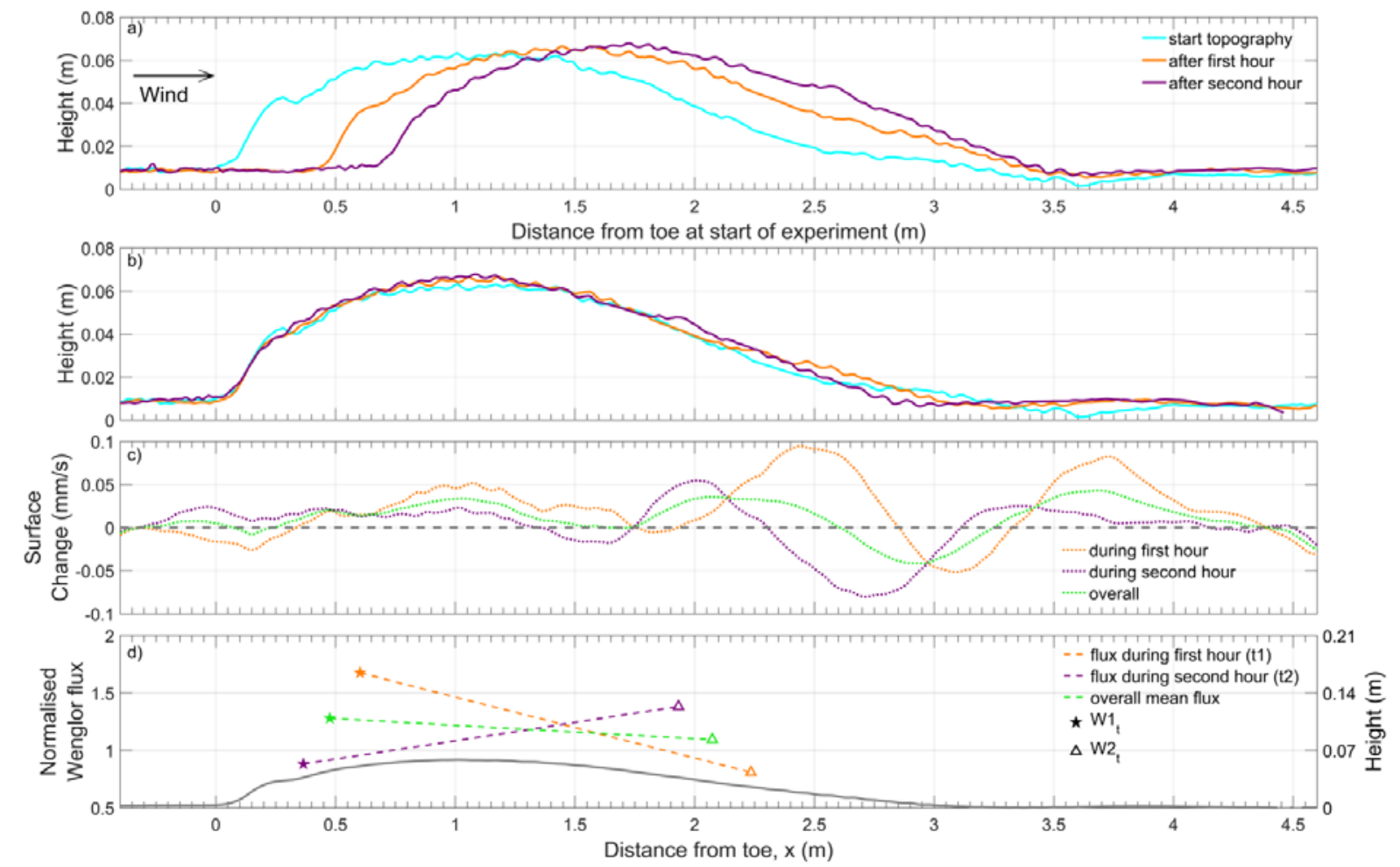

509

510 Figure 5: Protodune cross sections from transect Wx2 corresponding to the three

511 surfaces indicated in Figure 2 showing a) actual location at three points in time for

512 the unsmoothed data gridded at $0.01 \mathrm{~m}$ resolution, and b) offset horizontally by the

513 migration rate to reveal change in bedform morphology over time. c) Rate of surface

514 elevation change over differing periods showing deposition over the crestal area and

515 variation in erosion and deposition in the lee of the protodune. d) Variation in

516 normalised sand flux at W1 and W2, time-averaged for specific durations of the

517 experiment. The overall mean flux measurements upwind and downwind of the crest

518 indicate a reduction in transport (18\%) in line with the measured accretion over the

519 crest region (c). Topography (grey) in (d) is the time-averaged Wx2 cross section

520 shown in Figure 2b. 